Check for updates

Cite this: Chem. Commun., 2019, 55,1502

Received 7th November 2018,

Accepted 2nd January 2019

DOI: $10.1039 / c 8 c c 08879 a$

rsc.li/chemcomm

\section{A ratiometric fluorescent probe of methionine sulfoxide reductase with an improved response rate and emission wavelength $\dagger$}

\author{
Liangwei Zhang, (D) *ab Shoujiao Peng, (D) ac Jinyu Sun, ${ }^{a}$ Ruijuan Liu, ${ }^{a}$ Shudi Liu ${ }^{a}$ and \\ Jianguo Fang (iD *a
}

A ratiometric fluorescent probe of methionine sulfoxide reductase, Msr-Ratio, was disclosed for monitoring the enzyme activity in vitro and in live cells. The probe displayed favorable properties such as a nearly 400 -fold fluorescence change, fast response rate ( $<30 \mathrm{~min}$ ), large Stokes shift (120 nm), and green emission (550 nm).

Methionine sulfoxide reductase (Msr) enzymes are well known for their specific functions in protecting cells against oxidative damage caused by reactive oxygen species (ROS) and repairing methionine sulfoxide (MetO) residues in proteins/peptides by the reduction of MetO to methionine (Met). ${ }^{1-5}$ It is commonly known that MetO are present in two different oxidative forms, $S$-MetO epimer and $R$-MetO epimer, since the asymmetric center is the sulfur in MetO. Msr A and Msr B, two important members of the Msr family, can specifically catalyze the reduction of the $S$-MetO epimer and $R$-MetO epimer, respectively. ${ }^{6,7}$ The oxidative damage of Met protein residues would result in structural and functional changes in proteins that may implicate in aging-related neurodegenerative diseases such as Alzheimer's and Parkinson's diseases. ${ }^{8-10}$ Although Msr enzymes play important roles in repairing the structure and function of proteins, the methods used to directly monitor their activity in live cells are limited, ${ }^{11,12}$ which greatly hinders the exploration of their functions in physiological or pathological processes.

Fluorescent probes have been demonstrated to be indispensable tools in the field of biochemistry, clinical diagnosis, analytical, and environmental chemistry ${ }^{13-22}$ due to multiple advantages such as non-destructive detection, real-time monitoring, high sensitivity and

\footnotetext{
${ }^{a}$ State Key Laboratory of Applied Organic Chemistry and College of Chemistry and Chemical Engineering, Lanzhou University, Lanzhou 730000, China. E-mail: fangjg@lzu.edu.cn

${ }^{b}$ Key Laboratory of Coastal Environmental Processes and Ecological Remediation, Yantai Institute of Coastal Zone Research, Chinese Academy of Sciences, Yantai 264003, China. E-mail: liangweizhang@yic.ac.cn

${ }^{c}$ Department of Molecular Medicine, The Scripps Research Institute, 130 Scripps Way, Jupiter, FL 33458, USA

$\dagger$ Electronic supplementary information (ESI) available: Experimental details, original NMR and MS spectra and supplementary figures. See DOI: 10.1039/c8cc08879a
}

resolution, as well as low instrumental cost. The development of specific fluorescent probes for various analytes has experienced rapid growth in the past years. ${ }^{23-32}$ Up to now, only two paradigmatic fluorescent probes have been reported for monitoring Msr activity. ${ }^{11,12}$ The design of Msr fluorescent probes is now based on two important factors. First, recent studies demonstrated that Msr can not only reduce methionine sulfoxides, but also compounds equipped with a methyl sulfoxide group such as sulindac, dimethyl sulfoxide (DMSO), mesoridazine, sulmazole, TCBZSX, and sulforaphane. ${ }^{6}$ Second, the reduction of the electronwithdrawing methylsulfinyl group to the electron-donating methyl sulfide group can lead to a change in the emission signal via the intramolecular charge transfer (ICT) process, which is one of the most outstanding mechanisms for designing fluorescent probes. ${ }^{24,33-35}$ In our lab, we created a small probe library containing 23 dyes uniformly conjugated with a methyl sulfoxide group, from which, Msr-blue (4-methyl-7-(methylsulfinyl)$2 \mathrm{H}$-chromen-2-one, Scheme 1A) was identified as an off-on Msr fluorescent probe with a $>100$-fold increase in the emission intensity and has been applied for monitoring Msr activity in different cell lines. We also used Msr-blue to disclose the compromised Msr activity in a Parkinson's disease model. ${ }^{11}$ Dr Makukhin et al. used a chiral starting material to construct the optically pure probe, $(S)$-Sulfox-1, to monitor Msr A activity in bacterial cells. ${ }^{12}$ Both $(S)$-Sulfox-1 and Msr-blue still bear some limitations. For example, the emission wavelength of Msr-blue is short (438 nm), the reduction of the probe by Msr is slow ( $>2 \mathrm{~h}$ ), a large amount of enzyme is required to activate $(S)$-Sulfox-1, and there is only a weak enhancement in the fluorescence signal after $(S)$-Sulfox-1 reduction. Therefore, the
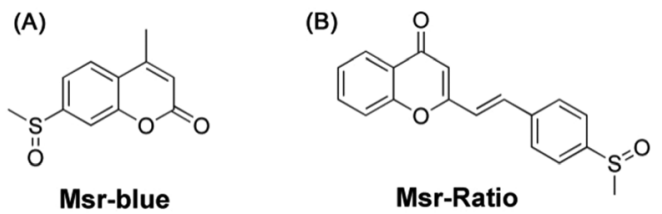

Scheme 1 The structures of the Msr probes, (A) Msr-blue and (B) Msr-Ratio. 
development of novel Msr probes with improved properties is urgently needed. Herein, we reported the synthesis and characterization of 2-(4-(methylsulfinyl)styryl)- $4 \mathrm{H}$-chromen-4-one (named Msr-Ratio and shown in Scheme 1B) as a ratiometric Msr fluorescent probe. The probe displays multiple favorable properties such as nearly 400 -fold fluorescence change, a fast response rate $(<30 \mathrm{~min})$, a large Stokes shift $(120 \mathrm{~nm})$, and green emission (550 nm). Msr-Ratio is able to image Msr activity in different types of live cells.

Msr-Ratio was synthesized in two steps according to the published procedures (Scheme S1, ESI $\dagger$ ). ${ }^{35,36}$ The structure of the probe and synthetic intermediates were fully characterized by ${ }^{1} \mathrm{H}-\mathrm{NMR},{ }^{13} \mathrm{C}-\mathrm{NMR}$ and MS (Fig. S1-S5, ESI $\dagger$ ). The purity of the Msr-Ratio probe was determined to be $99 \%$ by HPLC (Fig. S6, ESI $\dagger$ )

We first investigated the UV spectra of the Msr-Ratio probe and its sulfide form (compound 2, Fig. S7 and S8, ESI $\dagger$ ). We selected the maximum absorption band for the sulfide form at $375 \mathrm{~nm}$ as the excitation wavelength. We then investigated whether dithiothreitol (DTT) would cause interferences in the assay as some molecules with a methyl sulfoxide group can be directly reduced by DTT. ${ }^{11}$ Fortunately, there were no interferences under different DTT concentrations from $1 \mathrm{mM}$ to $20 \mathrm{mM}$ (Fig. S9, $\mathrm{ESI} \dagger$ ). We chose a $5 \mathrm{mM}$ DTT concentration for all assays, which was consistent with our previous work. ${ }^{11}$ To our delight, the detailed fluorescence spectral studies showed that Msr-Ratio displayed a ratiometric character towards Msr in the presence of DTT in TE buffer (50 mM Tris-HCl, $1 \mathrm{mM}$ EDTA buffer, $\mathrm{pH}=7.40$, $37{ }^{\circ} \mathrm{C}$ ). The time-dependent fluorescence response of Msr-Ratio to the enzyme is depicted in Fig. 1A. Msr-Ratio emitted a weak fluorescence at $430 \mathrm{~nm}$ (fluorescence quantum yield, $\Phi$, was too low to be detected). As the enzymatic reaction progressed, the emission at $430 \mathrm{~nm}$ slightly decreased while a new emission at $550 \mathrm{~nm}$ remarkably increased $(\Phi=5.19 \%)$. These findings are well-matched with the spectrum of its sulfide form (Fig. S10, ESI $\dagger$ ). The fluorescence spectra had a common intersection point at $457 \mathrm{~nm}$. For clear observation, the amplified image of the wavelength peak from 380 to $470 \mathrm{~nm}$ is shown in Fig. S11 (ESI $\dagger$ ). The ratio of $F_{550} / F_{430}$ changed from 0.134 to 51.4 with a 384-fold difference. Ratiometric fluorescent probes are superior to other probes that are merely based on fluorescence increases
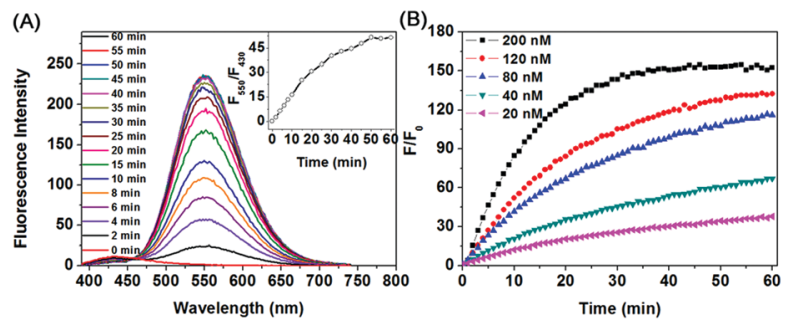

Fig. 1 Spectral changes of Msr-Ratio towards Msr/DTT in TE buffer. (A) Time course of Msr-Ratio $(10 \mu \mathrm{M})$ fluorescence changes in the presence of Msr A $(200 \mathrm{nM})$ and DTT (5 mM). Inset: Fluorescence changes of $F_{550} / F_{430}$. (B) Timeand dose-dependent fluorescence response of Msr-Ratio in the presence of DTT $(5 \mathrm{mM})$ and Msr A $(0-200 \mathrm{nM})$. F/Fo was determined $\left(\lambda_{\text {ex }}=375 \mathrm{~nm}\right.$ and $\left.\lambda_{\mathrm{em}}=550 \mathrm{~nm}\right)$. or decreases because they have two different emission wavelengths and are measured by the ratio of the fluorescence intensities, which can provide a built-in correction for environmental effects. ${ }^{37-39}$ The fluorescence emission intensity was also collected at various enzyme concentrations (0-200 nM) and is shown in Fig. 1B. The fluorescence reached saturation levels within 30 minutes when the probe was incubated with $200 \mathrm{nM}$ of the enzyme, indicating that the Msr-Ratio probe had a fast response rate. The detection limit for the probe was $2.07 \mathrm{nM}$ according to the reported method (Fig. S12, ESI $\dagger$ ). ${ }^{30}$ The fluorescent spectral changes demonstrated that the Msr-Ratio probe had a good fluorescence response towards Msr. This can be ascribed to the reduction of the methyl sulfoxide to the methyl sulfide group by the enzyme, which enabled the transformation of an electron withdrawing group to an electron donating group and thus enhanced the ICT process.

In order to further confirm the proposed mechanism, i.e., the fluorescence response was due to the conversion of the methyl sulfoxide group to the methyl sulfide group by the enzyme, we carried out HPLC assays of the reaction. As shown in Fig. 2A, Msr-Ratio was converted to its corresponding sulfide form by Msr. Additional HPLC assays were performed to monitor the time-dependent enzymatic reaction. We monitored the conversion of Msr-Ratio by incubating the probe with Msr and DTT in TE buffer at different time points $(5,10,20,40,80$, and $120 \mathrm{~min})$ as shown in Fig. 2B. After Msr-Ratio was incubated with Msr/DTT for 40 minutes, more than $40 \%$ of Msr-Ratio $(\sim 4.22 \mu \mathrm{M})$ converted to its corresponding sulfide form (more than 40\%, $\sim 4.19 \mu \mathrm{M}$ ), indicating a clean conversion of the probe to its sulfide form. Msr-Ratio was a racemic mixture containing both $S$-epimers and $R$ epimers. The enzyme used here was Msr A, which can only transform $S$-epimers into its sulfide form. The remaining amount of Msr-Ratio was about half the initial amount of Msr-Ratio, indicating that the probe may have been completely converted to
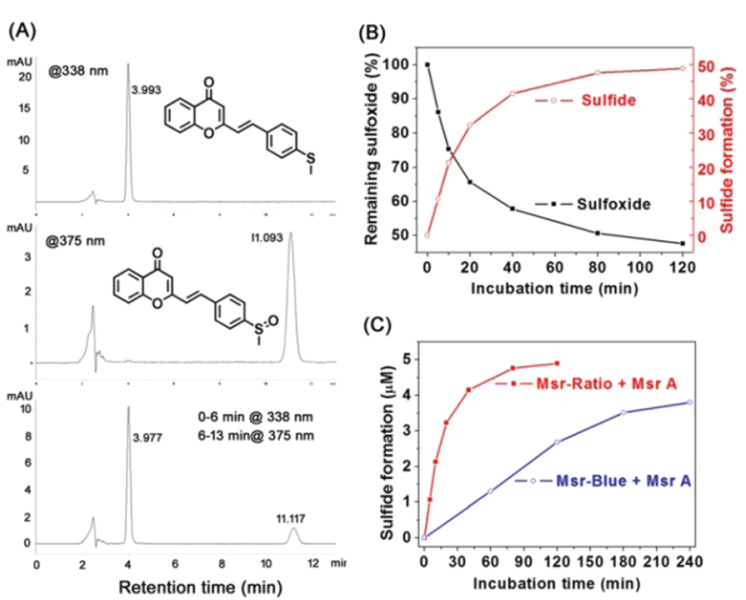

(C)

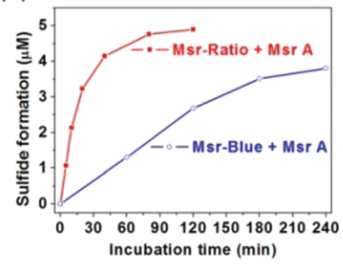

Fig. 2 (A) HPLC analysis of the Msr-Ratio conversion by Msr A/DTT in TE buffer. (B) Time course for the conversion of Msr-Ratio $(10 \mu \mathrm{M})$ to the corresponding sulfide form (compound 2) in the presence of Msr A $(200 \mathrm{nM})$ and DTT $(5 \mathrm{mM})$ by HPLC. (C) Comparison of the conversion of Msr-Ratio and Msr-blue in the presence of DTT (5 mM) and Msr A (200 nM) by HPLC. 
the sulfide form by the Msr enzymes. Subsequently, we performed additional HPLC assays to compare the response time for the enzymatic conversion of Msr-Ratio and Msr-blue under the same conditions. As illustrated in Fig. 2C, the Msr-Ratio showed a faster response time towards the enzyme compared to Msr-blue.

To verify the applications of the Msr-Ratio for the analysis of intracellular Msr activity, we performed selectivity experiments towards various reducing species. As shown in Fig. 3A, only Msr turned on the fluorescence with nearly a 101-fold increase. Under the same experimental conditions, none of the tested reducing agents turned on the fluorescence, including various small molecule thiols, glutathione reductase (GR), thioredoxin (Trx), thioredoxin reductase (TrxR), bovine serum albumin (BSA), ascorbate, and tris(2-carboxyethyl)phosphine (TCEP). Next, we examined the Msr activity in cell lysates from HL60 cells. In Fig. 3B and C, the fluorescence displayed a gradual enhancement during the incubation of Msr-Ratio with the lysate and DTT. DMSO and MetSO are well-known Msr inhibitors. ${ }^{40}$ The reduction of Msr-Ratio by the cell extract was inhibited by both
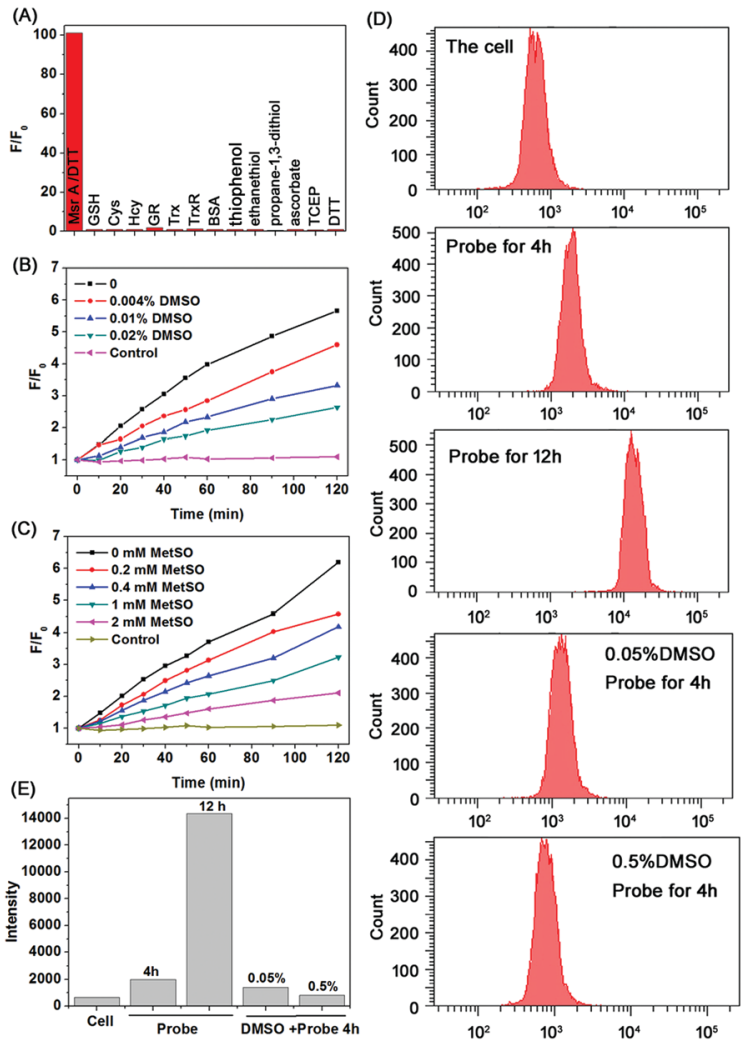

Fig. 3 Selective reduction of Msr-Ratio by Msr. (A) Fluorescence response of the Msr-Ratio probe $(10 \mu \mathrm{M})$ towards different analytes, including Msr A $(120 \mathrm{nM})$ with DTT $(5 \mathrm{mM})$ and small molecules $(1 \mathrm{mM})$ or proteins (20 $\mu \mathrm{g} \mathrm{mL}^{-1} \mathrm{GR}, 1 \mathrm{mg} \mathrm{mL}^{-1} \mathrm{BSA}, 0.2 \mathrm{mM} \mathrm{NADPH}, 80 \mu \mathrm{g} \mathrm{mL}^{-1} \mathrm{Trx}$, and $200 \mathrm{nM}$ TrxR), recorded at $0.5 \mathrm{~h}$. $F / F_{0}$ was determined $\left(\lambda_{\mathrm{ex}}=375 \mathrm{~nm}\right.$ and $\lambda_{\mathrm{em}}=550 \mathrm{~nm}$ ). Inhibition of the HL60 lysate-mediated Msr-Ratio reduction by DMSO (B) and MetSO (C). (D and E) Flow cytometric analysis of the monitored Msr cellular activity. Top to bottom: the cells, the cells incubated with the probe for $4 \mathrm{~h}$, the cells incubated with the probe for $12 \mathrm{~h}$, and the cells incubated with the probe for $4 \mathrm{~h}$ before pre-treatment with different amounts of DMSO $(0.05 \%$ and $0.5 \%)$.
DMSO and MetSO, suggesting that the reduction of Msr-Ratio was from Msr enzymes in the cell lysate. The corresponding spectra for Msr-Ratio treatment with the cell lysate in the presence of DMSO or MetSO are shown in Fig. S13 and S14 (ESI $\dagger)$, respectively. Compared to the treatment with pure Msr A, the emission wavelength was affected and the ratiometric character was no longer pronounced. This was mainly because the components of the cell lysate were intricate and had a fluorescence background. Results from the flow cytometric analysis reveal that Msr-Ratio can be effectively reduced by Msr enzymes in live cells. The time-dependent enhancement of the fluorescence intensity and its inhibition by DMSO are shown in Fig. 3D and E, respectively. All these results demonstrate that Msr-Ratio has a high selectivity for Msr and the potential to monitor Msr in live cells.

We further explored Msr-Ratio to visually observe Msr activity in live cells. As shown in Fig. 4, there was a very weak background signal in live HL60 cells after the addition of the probe. As the Msr-Ratio $(10 \mu \mathrm{M})$ incubation time increased in cells from 0 to $8 \mathrm{~h}$, the fluorescence signal constantly strengthened, which was consistent with the flow cytometric analysis results as shown in Fig. 3D. If the cells were treated with DMSO (2\%) for $0.5 \mathrm{~h}$ prior to the addition of Msr-Ratio, the fluorescence signal was almost completely inhibited. Herein, we note that a blue fluorescence was observed although the probe had a fluorescence response at $550 \mathrm{~nm}$. This result was mainly because under the light source excitation at $375 \mathrm{~nm}$, the fluorescence microscope could only collect a blue fluorescence imaging signal. The imaging of Msr activity in $293 \mathrm{~T}$ cells and its inhibition by DMSO were also demonstrated (Fig. S15, ESI $\dagger$ ). All these results suggest that Msr-Ratio is suitable for imaging cellular Msr activity.

To explore the application of Msr-Ratio in biological samples, we used the probe to measure the Msr activity in different organs of a mouse, including liver, spleen, kidney, brain and heart. The fluorescence assay is shown in Fig. 5A and Fig. S16 (ESI $\dagger$ ). The trend for the Msr activity was kidney $>$ liver $\gg$ heart $\sim$ brain $\sim$ spleen. These results matched well with the results from the western blot assay reported in our previous work. ${ }^{11}$ We selected the kidney lysate to measure the reduction of Msr-Ratio in the presence of the Msr inhibitors, DMSO and MetSO (Fig. S17 and S18, ESI $\dagger$ ), thus revealing the highly selective reduction of Msr-Ratio by Msr. To confirm the specific reduction of Msr-Ratio by Msr, we determined the reduction of Msr-Ratio with mouse kidney lysates before and after an Msr A immune-depletion. The removal of Msr A from the lysate significantly reduced the fluorescence intensity (Fig. 5B), demonstrating the essential role of Msr in the reduction of Msr-Ratio. The Msr-Ratio

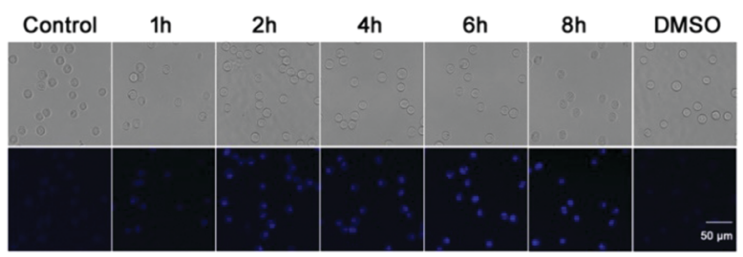

Fig. 4 Msr activity imaging in HL60 cells. 

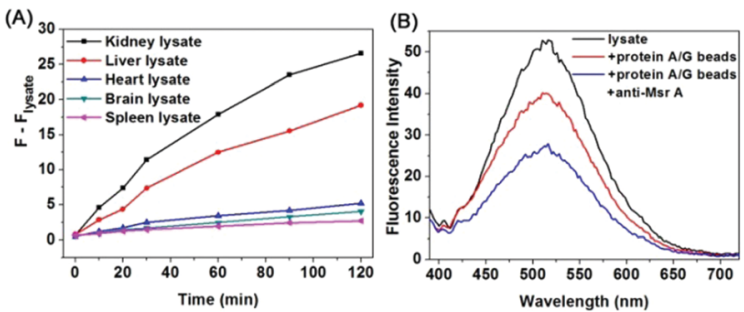

Fig. 5 (A) Fluorescence response of Msr-Ratio incubated with Msr activity in the organs of a mouse. (B) Msr-Ratio reduction by mouse kidney lysate before and after Msr A immunoprecipitation (IP).

reduction activity in the lysate after the immune-removal of Msr A is likely from Msr B and other isoforms of Msr, as the Msr A antibody does not cross-react with other Msr enzymes. Overall, the results support that Msr-Ratio is a specific fluorogenic substrate of Msr.

In conclusion, Msr-Ratio, a novel Msr fluorescent probe with a ratiometric characteristic and improved properties was developed. The probe demonstrated an outstanding capability for selectively monitoring the Msr activity. Msr-Ratio is able to determine the Msr activity in biological samples and image Msr functions in different types of live cells.

This work was financially supported by the Natural Science Foundation of China (21778026, 21778028, 21402077, 21572093, and 21701074) and the 111 Project. We appreciated Prof. Hong Yu from the School of Basic Medical Sciences at Wuhan University in China for the recombinant Msr A protein.

\section{Conflicts of interest}

There are no conflicts to declare.

\section{Notes and references}

1 H. Weissbach, L. Resnick and N. Brot, Biochim. Biophys. Acta, 2005, 1703, 203-212.

2 J. Moskovitz, S. Bar-Noy, W. M. Williams, J. Requena, B. S. Berlett and E. R. Stadtman, Proc. Natl. Acad. Sci. U. S. A., 2001, 98, 12920-12925.

3 A. Kaya, B. C. Lee and V. N. Gladyshev, Antioxid. Redox Signaling, 2015, 23, 814-822.

4 M. J. Davies, Biochim. Biophys. Acta, 2005, 1703, 93-109.

5 A. Hansel, S. H. Heinemann and T. Hoshi, Biochim. Biophys. Acta, 2005, 1703, 239-247.

6 C. Achilli, A. Ciana and G. Minetti, BioFactors, 2015, 41, 135-152.

7 S. Boschi-Muller, A. Gand and G. Branlant, Arch. Biochem. Biophys., 2008, 474, 266-273.

8 C. Schoneich, Biochim. Biophys. Acta, 2005, 1703, 111-119.

9 J. Moskovitz, Biochim. Biophys. Acta, 2005, 1703, 213-219.
10 A. B. Salmon, G. Kim, C. Liu, J. D. Wren, C. Georgescu, A. Richardson and R. L. Levine, Redox Biol., 2016, 10, 251-256.

11 L. Zhang, S. Peng, J. Sun, J. Yao, J. Kang, Y. Hu and J. Fang, Chem. Sci., 2017, 8, 2966-2972.

12 N. Makukhin, V. Tretyachenko, J. Moskovitz and J. Misek, Angew. Chem., Int. Ed., 2016, 55, 12727-12730.

13 W. Chen, S. Xu, J. J. Day, D. Wang and M. Xian, Angew. Chem., Int. Ed., 2017, 56, 16611-16615.

14 Y. Wi, H. T. Le, P. Verwilst, K. Sunwoo, S. J. Kim, J. E. Song, H. Y. Yoon, G. Han, J. S. Kim, C. Kang and T. W. Kim, Chem. Commun., 2018, 54, 8897-8900.

15 K. Singh, A. M. Rotaru and A. A. Beharry, ACS Chem. Biol., 2018, 13, 1785-1798.

16 S. Yoon, E. W. Miller, Q. He, P. H. Do and C. J. Chang, Angew. Chem., Int. Ed., 2007, 119, 6778-6781.

17 M. H. Lee, A. Sharma, M. J. Chang, J. Lee, S. Son, J. L. Sessler, C. Kang and J. S. Kim, Chem. Soc. Rev., 2018, 47, 28-52.

18 A. F. Schneider and C. P. Hackenberger, Curr. Opin. Biotechnol., $2017,48,61-68$.

19 X. Li, X. Gao, W. Shi and H. Ma, Chem. Rev., 2014, 114, 590-659.

20 Y. Yang, Q. Zhao, W. Feng and F. Li, Chem. Rev., 2013, 113, 192-270.

21 S. Pan, S.-Y. Jang, S. S. Liew, J. Fu, D. Wang, J.-S. Lee and S. Q. Yao, Angew. Chem., Int. Ed., 2018, 130, 588-592.

22 R. Zhu, S. Wang, Z. Xue, J. Han and S. Han, Chem. Commun., 2018, 54, 11566-11569.

23 K. Xu, D. Luan, X. Wang, B. Hu, X. Liu, F. Kong and B. Tang, Angew. Chem., Int. Ed., 2016, 55, 12751-12754.

24 L. Zhang, D. Duan, Y. Liu, C. Ge, X. Cui, J. Sun and J. Fang, J. Am. Chem. Soc., 2014, 136, 226-233.

25 Z. Liu, X. Zhou, Y. Miao, Y. Hu, N. Kwon, X. Wu and J. Yoon, Angew. Chem., Int. Ed., 2017, 56, 5812-5816.

26 H. W. Liu, L. Chen, C. Xu, Z. Li, H. Zhang, X. B. Zhang and W. Tan, Chem. Soc. Rev., 2018, 47, 7140-7180.

27 X. Chen, F. Wang, J. Y. Hyun, T. Wei, J. Qiang, X. Ren, I. Shin and J. Yoon, Chem. Soc. Rev., 2016, 45, 2976-3016.

28 M. H. Lee, H. M. Jeon, J. H. Han, N. Park, C. Kang, J. L. Sessler and J. S. Kim, J. Am. Chem. Soc., 2014, 136, 8430-8437.

29 H. Xiao, C. Wu, P. Li, W. Gao, W. Zhang, W. Zhang, L. Tong and B. Tang, Chem. Sci., 2017, 8, 7025-7030.

30 Y. Hu, J. Kang, P. Zhou, X. Han, J. Sun, S. Liu, L. Zhang and J. Fang, Sens. Actuators, B, 2018, 255, 3155-3162.

31 J. M. Goldberg, F. Wang, C. D. Sessler, N. W. Vogler, D. Y. Zhang, W. H. Loucks, T. Tzounopoulos and S. J. Lippard, J. Am. Chem. Soc., 2018, 140, 2020-2023.

32 B. Shi, X. Gu, Q. Fei and C. Zhao, Chem. Sci., 2017, 8, 2150-2155.

33 Z. R. Dai, G. B. Ge, L. Feng, J. Ning, L. H. Hu, Q. Jin, D. D. Wang, X. Lv, T. Y. Dou, J. N. Cui and L. Yang, J. Am. Chem. Soc., 2015, 137, 14488-14495.

34 M. H. Lee, J. H. Han, J. H. Lee, H. G. Choi, C. Kang and J. S. Kim, J. Am. Chem. Soc., 2012, 134, 17314-17319.

35 W. Sun, J. Fan, C. Hu, J. Cao, H. Zhang, X. Xiong, J. Wang, S. Cui, S. Sun and X. Peng, Chem. Commun., 2013, 49, 3890-3892.

36 D. Yu, F. Huang, S. Ding and G. Feng, Anal. Chem., 2014, 86, 8835-8841.

37 Y. Chen, C. Zhu, Z. Yang, J. Chen, Y. He, Y. Jiao, W. He, L. Qiu, J. Cen and Z. Guo, Angew. Chem., Int. Ed., 2013, 52, 1688-1691.

38 Q. Wan, S. Chen, W. Shi, L. Li and H. Ma, Angew. Chem., Int. Ed., 2014, 53, 10916-10920.

39 X. Jia, Q. Chen, Y. Yang, Y. Tang, R. Wang, Y. Xu, W. Zhu and X. Qian, J. Am. Chem. Soc., 2016, 138, 10778-10781.

40 D. Brunell, H. Weissbach, P. Hodder and N. Brot, Assay Drug Dev. Technol., 2010, 8, 615-620. 